\title{
"Primary" and "secondary" muscle-invasive bladder cancer is more than just a surrogate for molecular subtypes
}

\author{
Eugene J. Pietzak, Hikmat Al-Ahmadie, Aleksandra Walasek, Dean F. Bajorin, Jonathan E. Rosenberg, \\ Bernard H. Bochner, David B. Solit, Gopa Iyer \\ Memorial Sloan Kettering Cancer Center, New York, NY, USA \\ Correspondence to: Eugene J. Pietzak. Urology Service, Department of Surgery, Kimmel Center for Prostate and Urologic Cancers, Memorial Sloan \\ Kettering Cancer Center, 353 East 68th Street, New York, NY 10065, USA. Email: pietzake@MSKCC.org.
}

Submitted Jul 31, 2019. Accepted for publication Dec 03, 2019.

doi: $10.21037 /$ tcr.2019.12.21

View this article at: http://dx.doi.org/10.21037/tcr.2019.12.21

We would like to thank Drs. Szarvas, Olah, and Reis for their thoughtful and insightful comments regarding our recent publication on patients who initially present with muscle-invasive bladder cancer ("primary" MIBC) compared to patients with non-muscle-invasive bladder cancer (NMIBC) who unfortunately experience progression to MIBC ("secondary" MIBC). As this latter group account for only $15-20 \%$ of MIBC patients, such patients were underrepresented in the clinical trials from which guidelines for neoadjuvant chemotherapy were based.

To address the possibility that patients with secondary MIBC might respond to neoadjuvant chemotherapy differently than patients with primary" MIBC, we performed a retrospective analysis that integrated tumor genomic profiling data generated at MSKCC and by the Tumor Cancer Genome Network with patient demographic and treatment response data. We observed that the pathologic response rate to neoadjuvant chemotherapy was lower among patients with secondary MIBC than patients with primary MIBC on both univariable (26\% vs. $45 \%$, $\mathrm{P}=0.02$ ) and multivariable analyses adjusted for age, sex, and tumor stage (odds ratio $=0.4 ; 95 \%$ confidence interval: 0.18 $0.84, \mathrm{P}=0.02)$ (1). Further, we found that secondary MIBC was associated with significantly worse recurrence-free survival (RFS), cancer-specific survival (CSS), and overall survival (OS) compared to primary MIBC. Unexpectedly, we found that patients with secondary MIBC who had received neoadjuvant chemotherapy had worse oncologic outcomes than patients with secondary MIBC treated with immediate cystectomy.

We next analyzed the mutational profiles of the two groups to determine whether differences in the pattern of oncogenic drivers could explain the lack of sensitivity of secondary MIBC to platinum-based neoadjuvant chemotherapy. Among mutations previously implicated in chemotherapy response, only ERCC2 missense mutations were significantly enriched in the primary versus secondary MIBC tumors ( $11 \%$ vs. $1.8 \%, \mathrm{P}=0.044)$. We then sought to validate these findings in an independent cohort of prospectively sequenced MIBC tumors from chemotherapynaïve patients treated at MSK. Again, we found that ERCC2 missense mutations were significantly enriched in primary versus secondary MIBC specimens $(17.1 \%$ vs. $0 \%, \mathrm{P}=0.033)$ in this prospectively enrolled cohort. In the combined analysis of all patients, ERCC2 missense mutations were significantly more common in primary versus secondary MIBC tumors [12\% (48/400) vs. $1.3 \%$ (1/79), $\mathrm{P}=0.004]$. There are limitations to all retrospective studies, and we strongly believe that prospective validation in intentionto-treat cohorts is required before a change in clinical guidelines, but our observation of disparate treatment outcomes between primary and secondary MIBC raises several testable hypotheses while we await prospective validation.

NMIBC is a heterogenous disease. While Szarvas et al. note that tumor mutational burden is lower in noninvasive versus MIBC, this applies mainly to low-grade tumors. Our group has reported on the genomic profile of over 100 NMIBC tumors using MSK-IMPACT, a targeted exon-capture next-generation sequencing platform (2). This analysis found a high rate of DNA damage repair gene mutations and high overall tumor mutational burden in high-grade NIMBC. The results suggest that low and high grade NMIBC have distinct genomic profiles with 
high grade non-invasive tumors more closely resembling MIBC. The most common alterations in DNA repair pathways were missense mutations in ERCC2, occurring in $17 \%$ of high-grade NMIBC. The abundance of ERCC2 mutations in pre-treatment high-grade NMIBC, along with the near absence of ERCC2 mutations in secondary $M I B C$, raises the question of whether tumor neoantigen driven clonal evolution under the selective pressure of BCG immunotherapy may contribute to BCG responsesimilar to what has been reported with immune checkpoint inhibition in some tumor types with high tumor mutational burden. Our group is testing this hypothesis in both a prospective and retrospective fashion to determine if deleterious DNA damage repair gene mutations, tumor mutational burden, and/or tumor neoantigen burden can serve as predictive biomarkers for BCG response. While we believe clonal selection from BCG immunotherapy may be an important mechanism that contributes to the differences in neoadjuvant chemotherapy sensitivity observed in our study, most NMIBCs and MIBCs do not harbor a somatic DNA damage repair gene alteration. Further investigations into germline and epigenetic factors that contribute to the variation among patients are thus also underway by our group and others.

To determine if differences in molecular subtypes might also account for the observed differences in primary and secondary MIBC, we analyzed RNA expression data generated by the TCGA for the patients included in our discovery cohort. We found no difference in basal $v s$. luminal subtypes using either the TCGA $\mathrm{k}=5$ or BASE47 classifers. While we were unable to identify any enrichment of the luminal molecular subtype in secondary MIBC tumors, this does not imply that we are accepting the null hypothesis that no difference in subtypes exist. With the UROMOL NMIBC study suggesting a higher prevalence of luminal tumors among patients who experienced progression to secondary MIBC (so called "group 2" tumors), it is certainly possible that a difference between subtypes could emerge between primary and secondary MIBC tumors upon completion of larger transcriptomic profiling studies (3). As the molecular subtypes of NMIBC are not as well defined as those of MIBC, it is unknown whether molecular subtypes will prove to be predictive of BCG response or progression to secondary MIBC in BCG-treated patients, or whether molecular subtypes may change following exposure to BCG treatment. To explore this possibility, our group is currently performing whole transcriptomic RNA sequencing on pre- and post-BCG tumors from patients who developed secondary MIBC.

It is also important to acknowledge the limitations of molecular subtyping with regards to tumor heterogeneity and plasticity, especially since the clinical utility of molecular subtyping remains unknown. None of the proposed MIBC subtype classifiers have yet to be prospectively validated, but several studies suggest that patients with luminal tumors are less likely to benefit from neoadjuvant chemotherapy as compared to those with basal tumors $(4,5)$. Yet these same studies also suggest that patients with luminal tumors tend to have a better prognosis as compared to those with basal tumors regardless of whether neoadjuvant therapy was administered. This is inconsistent with our observations that patients with secondary MIBC have worse oncologic outcomes (1). Therefore, even if secondary MIBC does turn out to be enriched with luminal tumors, this is unlikely to fully explain the differences in response to NAC among patients with primary and secondary MIBC.

We do agree with Szarvas et al. that important differences between our discovery cohort and validation cohorts may exist and that further investigation into the molecular subtypes of primary and secondary MIBC is needed. Specifically, we acknowledge that it is a limitation of our study that RNA expression data were available only for the patients in the publicly available bladder TCGA cohort. Notably, we had to be less rigorous in our classification of primary and secondary MIBC for TCGA specimens collected at other institutions, with primary MIBC defined as "no history of NMIBC," whereas secondary MIBC was defined as "a history of NMIBC and reported to have received prior BCG" (1). This is in contrast to MSK patients in both our discovery and validation cohorts who were considered to have secondary MIBC only if they had an initial non-muscle invasive tumor (Tis, Ta, or T1 with uninvolved muscularis propria in their specimen) that was confirmed by a re-staging transurethral resection of the bladder tumor (TURBT) or at least one follow-up cystoscopy prior to the eventual diagnosis of MIBC (1). Given our inability to review the charts from the non-MSK patients in the TCGA cohort, we were unable to control for potential under-staging that might have occurred in patients who were treated with BCG without a re-staging TURBT or other potential mitigating factors that could result in inadequate or delayed treatment.

Ultimately prospective validation of the clinical and genomic differences between primary and secondary MIBC observed in our study in an intention-to-treat cohort is required. We believe this will be possible using the genomic 
and transcriptomic data generated within the context of the Southwest Oncology Group (SWOG) COXEN study (NCT02177695) which is investigating the association between multiple biomarkers and response to neoadjuvant chemotherapy, as well as through the Alliance for Clinical Trials in Oncology bladder-sparing trial (NCT03609216) that seeks to avoid cystectomy in patients with select DNA damage repair gene alterations (e.g., ERCC2) if they achieve clinical downstaging following cisplatin-based neoadjuvant chemotherapy.

\section{Acknowledgments}

Funding: This work was supported by the Sidney Kimmel Center for Prostate and Urologic Cancers, the Michael and Zena Wiener for Therapeutics Program in Bladder Cancer, Pin Down Bladder Cancer, Cycle for Survival, the Marie-Josee and Henry R. Kravis Center for Molecular Oncology, NIH/NCATS Grant Number UL1-TR002384, the National Cancer Institute Cancer Center Core Grant Number P30-CA008748, MSKCC SPORE in Bladder Cancer P50- CA221745, NIH/NCI K12 Paul Calabresi Career Development Award for Clinical Oncology (K12 CA184746).

\section{Footnote}

Conflicts of Interest: EJ Pietzak: Scientific Advisory Board: Merck; Consultant: Chugai Pharma. H Al-Ahmadie: Consultant: Bristol-Myers-Squibb, AstraZeneca, EMD Serono. DF Bajorin: Honoraria from the speaker's bureau of Merck; Consultant/advisory board: Merck, Pfizer, BristolMyers Squibb, Urogen, Genentech,Eli Lilly. JE Rosenberg: Stock and Other Ownership Interests: Merck, Illumina; Honoraria: UpToDate, Bristol-Myers Squibb, AstraZeneca, Medscape, Vindico, Peerview, Chugai Pharma; Consulting or Advisory Role: Lilly, Merck, Agensys, Roche/ Genentech, Sanofi, AstraZeneca/MedImmune, BristolMyers Squibb, EMD Serono, Seattle Genetic, Bayer, InovioPharmaceuticals, BioClin Therapeutics, QED Therapeutic, Adicet Bio,Sensei Biotherapeutics; Patents, Royalties, Other Intellectual Property: Predictor of platinum sensitivity; Research Funding: Genentech, Oncogenex, Agensys, Mirati Therapeutics, Novartis, Viralytics, Genen-tech/Roche, Incyte, Seattle Genetics, Bayer; Travel, Accommodations, Expenses: Genentech/ Roche, Bristol-Myers Squibb. BH Bochner: Chair of data safety monitoring committee: Genentech; CourseInstructor:
Olympus. DB Solit: Consultant for Pfizer, Loxo Oncology and Illumina. G Iyer: Consultant: Bayer.

Ethical Statement: The authors are accountable for all aspects of the work in ensuring that questions related to the accuracy or integrity of any part of the work are appropriately investigated and resolved.

Open Access Statement: This is an Open Access article distributed in accordance with the Creative Commons Attribution-NonCommercial-NoDerivs 4.0 International License (CC BY-NC-ND 4.0), which permits the noncommercial replication and distribution of the article with the strict proviso that no changes or edits are made and the original work is properly cited (including links to both the formal publication through the relevant DOI and the license). See: https://creativecommons.org/licenses/by-nc-nd/4.0/.

\section{References}

1. Pietzak EJ, Zabor EC, Bagrodia A, et al. Genomic Differences Between "Primary" and "Secondary" Muscleinvasive Bladder Cancer as a Basis for Disparate Outcomes to Cisplatin-based Neoadjuvant Chemotherapy. Eur Urol 2019;75:231-9.

2. Pietzak EJ, Bagrodia A, Cha EK, et al. Next-generation Sequencing of Nonmuscle Invasive Bladder Cancer Reveals Potential Biomarkers and Rational Therapeutic Targets. Eur Urol 2017;72:952-9.

3. Hedegaard J, Lamy P, Nordentoft I, et al. Comprehensive Transcriptional Analysis of Early-Stage Urothelial Carcinoma. Cancer Cell 2016;30:27-42.

4. Choi W, Porten S, Kim S, et al. Identification of distinct basal and luminal subtypes of muscle-invasive bladder cancer with different sensitivities to frontline chemotherapy. Cancer Cell 2014;25:152-65.

5. Seiler R, Ashab HAD, Erho N, et al. Impact of Molecular Subtypes in Muscle-invasive Bladder Cancer on Predicting Response and Survival after Neoadjuvant Chemotherapy. Eur Urol 2017;72:544-54.

Cite this article as: Pietzak EJ, Al-Ahmadie H, Walasek A, Bajorin DF, Rosenberg JE, Bochner BH, Solit DB, Iyer G. "Primary" and "secondary" muscle-invasive bladder cancer is more than just a surrogate for molecular subtypes. Transl Cancer Res 2019;8(Suppl 6):S642-S644. doi: 10.21037/ tcr.2019.12.21 